\title{
Calculation of the Normalization Factor of Incomplete Pole Figures by Cubic Extrapolation
}

\author{
M. DAHMS, H.-J. BUNGE \\ Institut für Metallkunde und Metallphysik der TU Clausthal; Großer Bruch \\ 23; D-3392 Clausthal-Zellerfeld
}

(Received October 1, 1985; in final form December 10, 1985)

\begin{abstract}
The calculation of orientation distribution functions from incomplete pole figures can be carried out by a least squares aproximation of the texture coefficients $\mathrm{C}_{l}^{\mu \nu}$ and the normalization factors $\mathbf{N}_{h k l}$ to the available experimental data. This procedure is less susceptable to instabilities due to experimental errors if the normalization factors can be calculated independently of the coefficients $C_{l}^{\mu \nu}$. In the case of cubic materials, the relationship $\mathrm{F}_{2}^{0}=0$ to be fulfilled by pole figure values provides an independent condition for the calculation of the normalization factor. This condition can still be improved by taking the slopes of the pole density curves at $\alpha=\alpha_{\max }$ and $\alpha=90^{\circ}$ into account. An economic way to consider the slope in the pole figures is to use a cubic spline interpolation.
\end{abstract}

\section{CALCULATION OF THE ODF FROM COMPLETE POLE FIGURES}

The texture of a polycrystalline material is described by the orientation distribution function $f(g)$ which is usually calculated from experimentally determined pole figures $P_{h k l}(\alpha \beta)$ where $\alpha \beta$ are the spherical coordinates in a sample fixed coordinate system of the direction normal to the reflecting lattice plane $(h k l)$. The pole figures are defined as continuous distribution functions on the complete orientation sphere. Experimentally determined pole figures are, however, obtained only at certain discrete positions $\alpha_{i} \beta_{j}$ or integrated through intervals $\Delta \beta_{i}$ and they are often available only in an incomplete range of the orientation sphere. 
These two restrictions have to be taken into account when a texture function $f(g)$ is calculated numerically as a best fit to the available experimental data.

The ODF may be represented in terms of a series expansion. In the case of a cubic crystal and orthorhombic sample symmetry it has the form

$$
f(g)=\sum_{l=0}^{l \max } \sum_{\mu=1}^{M(l)} \sum_{\nu=1}^{N(l)} C_{l}^{\mu \nu} \ddot{T}_{l}^{\mu \nu}(g)
$$

where $\ddot{T}_{l}^{\mu \nu}(g)$ are harmonic functions of cubic-orthorhombic symmetry, $g$ represents the crystal orientation with respect to a sample fixed coordinate system. $C_{l}^{\mu v}$ are the unknown coefficients which are related to the pole figure coefficients $F_{l}^{v}$ by

$$
F_{l}^{v}(h k l)=\frac{4 \pi}{2 l+1} \sum_{\mu=1}^{M(l)} C_{l}^{\mu \nu} \dot{\dot{K}}_{l}^{\mu} \quad\left(\theta_{h k l} \gamma_{h k l}\right)
$$

where $\theta_{h k l}$ and ${ }_{y h k l}$ are spherical polar coordinates of the normal direction to the reflecting lattice plane referred to a crystal-fixed coordinate system and $\dot{K}_{l}^{\mu}$ are spherical harmonics of the cubic crystal symmetry. The pole figures are then expressed in terms of harmonic functions $\dot{K}_{l}^{\mu}$ of the orthorhombic sample symmetry

$$
I_{h k l}(\alpha \beta)=\frac{1}{N_{h k l}} \cdot \sum_{l=0}^{l \max } \sum_{\nu=1}^{F(l)} F_{l}^{v}(h k l) \cdot \dot{K}_{l}^{v}(\alpha \beta)
$$

where $I_{h k l}(\alpha \beta)$ is the measured intensity and $N_{h k l}$ is the corresponding normalization factor. The coefficients $F_{l}^{v}(h k l)$ can be obtained from the experimental data i.e. $I_{h k l}(\alpha \beta)$ by the inversion of eq. 3 .

$$
F_{l}^{v}(h k l)=N_{h k l} .8 . \int_{\alpha=0}^{\pi / 2} \int_{\beta=0}^{\pi / 2} I_{h k l}(\alpha \beta) . \dot{K}_{l}^{v}(\alpha \beta) \sin \alpha d \alpha d \beta
$$

In eq. 3 , the orthorhombic symmetry of the pole figure has already been taken into account.

The orthorhombic surface harmonic $\dot{K}_{l}^{v}(\alpha \beta)$ can be expressed in the form

$$
\dot{K}_{l}^{v}(\alpha \beta)=\frac{\varepsilon^{v}}{\sqrt{2 \pi}} \cos v \beta \sum_{s=0(2)}^{l} \alpha_{l}^{\prime v s} \cos s \alpha, \quad \varepsilon^{v}=\left\{\begin{array}{l}
1, v=0 \\
\sqrt{2}, v \neq 0
\end{array}\right.
$$

Using eq. 5 , the coefficients $F_{l}^{v}$ of eq. 4 can be expressed in the form 


$$
F_{l}^{v}(h k l)=N_{h k l} \cdot \frac{8 \varepsilon^{v}}{\sqrt{2 \pi}} \int_{\alpha=0}^{\pi / 2}\left[S_{h k l}^{v}(\alpha) \sin \alpha\right] \sum_{s=0(2)}^{l} a_{l}^{\prime} \cos \alpha d \alpha
$$

where

$$
S_{h k l}^{v}(\alpha)=\int_{\beta=0}^{\pi / 2} I_{h k l}(\alpha \beta) \cos v \beta d \beta
$$

are integrals over the angle $\beta$ only.

Two different experimental techniques are commonly used to measure $I_{h k l}(\alpha \beta)$. At a fixed angle $\alpha=\alpha_{i}$ the intensity can either be measured at fixed angles $\beta=\beta_{j}$ or it can be integrated through the interval from $\beta_{j}-1 / 2 \Delta \beta$ to $\beta_{j}+1 / 2 \Delta \beta$. In both cases values of $I_{h k l}(\alpha \beta)$ are available at the points $\alpha_{i} \beta_{j}$. In order to increase the accuracy of the calculation of $S_{h k l}^{v}(\alpha)$ the intensities can be linearly interpolated in smaller steps $\Delta \beta^{\prime}=1 / \mathrm{n} \Delta \beta$. The integration can then be carried out in the form

$$
S_{h k l}^{v}\left(\alpha_{i}\right)=\sum_{j=1}^{j \max } I_{h k l}\left(\alpha_{i} \beta_{j}\right) \int_{\beta=\beta_{j}-\frac{1}{2} \Delta \beta^{\prime}}^{\beta_{j}+\frac{1}{2} \Delta \beta^{\prime}} \cos v \beta d \beta
$$

were the integrals are solved analytically.

$S_{h k l}^{v}(\alpha)$ is then available at the points $\alpha_{i}$. In order to carry out the integration over $\alpha$ in eq. 6 , the expression in brackets can be interpolated by cubic splines. They consist of a number of cubic polynomial segments joined end to end with continuity in first and second derivatives at the joins, the coefficients of which can be calculated from the available values at the points $\alpha_{i}$ solving a set of linear equations. (For further details of the mathematical procedure and the corresponding subroutines see for example Jordan-Engeln and Reutter (1981)). Equation (6) can then be expressed in the form

$$
\begin{gathered}
F_{l}^{v}(h k l)=N_{h k l} \frac{8 \varepsilon^{v}}{\sqrt{2 \pi}} \sum_{i=1}^{i \max -1} \sum_{s=0(2)}^{l} a_{l}^{\prime v s} \\
\cdot \int_{\alpha=\alpha_{i}}^{\alpha_{i+1}}\left[A_{i}^{v}+B_{i}^{v}\left(\alpha-\alpha_{i}\right)+C_{i}^{v}\left(\alpha-\alpha_{i}\right)^{2}+D_{i}^{v}\left(\alpha-\alpha_{i}\right)^{3}\right] \cos s \alpha d \alpha
\end{gathered}
$$

With the coefficients of the cubic polynominals. $A_{i}^{v}$ to $D_{i}^{v}$ given, the integral can be solved analytically. In eq. 9 the normalization factor 
$N_{h k l}$ is usually unknown. It can, however, be calculated from the normalization condition

$$
F_{0}^{0}(h k l)=\sqrt{4 \pi}
$$

\section{INCOMPLETE POLE FIGURES}

In the case of incomplete pole figures the intensities $I_{h k l}(\alpha \beta)$ are available only in a restricted range $0 \leqq \alpha \leqq \alpha_{\max }$ whereas the range of $\beta$ is usually not restricted. In this case the coefficients $F_{l}^{v}(h k l)$ cannot be calculated from eq. 4 . The coefficients $C_{l}^{\mu v}$ can, however, be calculated directly from the measured intensity values by a least squares approximation

$$
\begin{gathered}
\sum_{(h k l)} w_{h k l} \int_{\alpha=0}^{\alpha_{\max }} \int_{\beta=0}^{\pi / 2}\left\{N_{h k l} \cdot I_{h k l}(\alpha \beta)-\sum_{l=0}^{l \max } \sum_{\mu=1}^{M(l)} \sum_{\nu=1}^{N(l)}\right. \\
\frac{4 \pi}{2 l+1} \cdot C_{l}^{\mu \nu} \dot{K}_{l}^{* \mu}(h k l) \dot{K}_{l}^{\nu}(\alpha \beta\}^{2} \sin \alpha d \alpha d \beta=\text { Min }
\end{gathered}
$$

in which the coefficients $C_{l}^{\mu v}$ and the normalization factors $N_{h k l}$ are unknown. The weight factors $w_{h k l}$ can, in most cases, be chosen as unity.

Two different approaches are then possible to solve eq. 11:

1. The normalization factors $N_{h k l}$ can be calculated from eq. 11 together with the coefficients $C_{1}^{\mu \nu}$.

2. The normalization factors $N_{h k l}$ can be calculated separately. Eq. 11 can then be solved with given values of $N_{h k l}$.

The first method, proposed by Bunge (1969), was used by Jura and Pospiech (1974) and by Morris (1975). For the second method, several variants have been suggested. Kern and Bergmann (1979) calculated a quasi-normalization. This method was improved by van Houtte (1980) using an iterative procedure. Liang et al. $(1981,1983)$ integrated eq. 11 at first over $\beta$ in order to determine $N_{h k l}$ and recently van Houtte (1984) used an approximation condition leading to a non linear system of equations. Humbert and Bergmann (1980) took into account that for cubic materials $F_{2}^{0}=0$ for all pole figures. This allows an estimation of the missing part of the corresponding integral in eq. 4.

It will be shown in this paper that this latter method can be further improved taking into account all available information about the 
missing part of the function $S_{h k l}^{0}(\alpha) \sin \alpha$. Besides the integral value $F_{2}^{0}=0$ used by Humbert and Bergmann this is the functional value and slope of this function at $\alpha=\alpha_{\max }$ and from symmetry reasons the slope at $\alpha=90^{\circ}$. This allows the determination of four parameters and hence a third order approximation to $S_{h k l}^{0}(\alpha) \sin \alpha$. If we split the integral eq. 3 into the measured and unmeasured range it follows from $F_{2}^{0}=0$ that

$$
\begin{aligned}
\int_{\alpha=0}^{\alpha} \int_{\beta=0}^{\pi / 2} I_{h k l}(\alpha \beta) \bar{P}_{2}(\alpha) \sin \alpha d \alpha d \beta \\
+\int_{\alpha=\alpha}^{\pi / 2} \int_{\max }^{\pi / 2} I_{h k l}(\alpha \beta) \bar{P}_{2}(\alpha) \sin \alpha d \alpha d \beta
\end{aligned}
$$

The first integral in eq. 12 can be calculated by the spline interpolation of eq. 9 with the appropriate value of $i_{\max }$. Using the definition of eq. 7 , the second integral contains the function $S_{h k l}^{0}(\alpha) \sin \alpha$ which is, however, unknown since $I_{h k l}(\alpha \beta)$ is unknown in this range. The unknown function can be approximated by a cubic polynominal.

$$
S_{h k l}^{0}(\alpha) \sin \alpha=A+B \alpha+C \alpha^{2}+D \alpha^{3}=W(\alpha)
$$

the coefficients of which are obtained from the boundary conditions at $\alpha=\alpha_{\max }$ and $\alpha=\pi / 2$

$$
\begin{aligned}
W\left(\alpha_{\max }\right) & =S_{h k l}^{0}\left(\alpha_{\max }\right) \sin \alpha_{\max } \\
W^{\prime}\left(\alpha_{\max }\right) & =\left[S_{h k l}^{0}\left(\alpha_{\max }\right) \sin \alpha_{\max }\right. \\
W^{\prime}(\pi / 2) & =0
\end{aligned}
$$

Where $S_{h k l}^{0}\left(\alpha_{\max }\right)$ is the value corresponding to the experimentally determined range of $I_{h k l}(\alpha \beta)$ and $\mathrm{W}^{\prime}$ is the first derivative of $\mathrm{W}(\alpha)$.

The first two equations require that the extrapolation polynominal continues the measured one with the same slope whereas the third one results from the symmetry condition at $\alpha=\pi / 2$. Furthermore the integral over the unmeasured range from $\alpha_{\max }$ to $\pi / 2$ is fixed by eq. 12 . This provides four equations from which the four coefficients $A$ to $D$ can be calculated. With both the measured and extrapolated functions $S_{h k l}^{0}(\alpha) \sin \alpha$, the normalization factor $N_{h k l}$ is then obtained from eq. 10 with eq. 9. Equation 11 can then be solved with known normalization factors $N_{h k l}$. (Note, that the extrapolation eq. 13 is only used to determine 
the normalization factor $N_{h k l}$. The extrapolated function does not explicitly enter eq. 11 from which the coefficients $C_{l}^{\mu v}$ are determined.)

In order to apply this method, the slope of the measured function at $\alpha=\alpha_{\max }$ is needed. It is thus essential to use the spline interpolation which determines this slope not only from the last interval $\alpha_{\max -1}$ to $\alpha_{\max }$ but with all the intensity values of the whole range from $\alpha=0$ to $\alpha=\alpha_{\max }$.

\section{RESULTS}

In order to test the proposed method, four synthetic textures were used, i.e. two (100)-fibre textures with $12.5^{\circ}$ and $7.5^{\circ}$ spread respectively and two (001)[100] - cube textures with the same spreads. The coefficients $C_{l}^{\mu v}\left(g_{0}, \omega_{0}\right)$ of a texture consisting of a single ideal orientation at $g_{0}$ with a gaussian spread $\omega_{0}$ are given by the expression

$$
C_{l}^{\mu \nu}\left(g_{0}, \omega_{0}\right)=\frac{\exp \left(-\frac{1}{4} l^{2} \omega_{0}^{2}\right)-\exp \left(-\frac{1}{4}(l+1)^{2} \omega_{0}^{2}\right)}{1-\exp \left(-\frac{1}{4} \omega_{0}^{2}\right)} \dot{T}_{l}^{\mu \nu}(g)
$$

The fibre textures are obtained with the coefficients of eq. 15 with $v=0$. In order to test the interpolation and extrapolation methods of the pole figures, only the coefficients $C_{l}^{\mu v}$ of even orders $l$ are needed. Hence, 'theoretical' textures were calculated according to eq. 1 with coefficients of even order $l$ from eq. 15 with $l_{\max }=34$. With eq. 2 , pole figure coefficients $F_{l}^{v}(h k l)$ and hence pole figures themselves were then obtained in steps of $\Delta \alpha=5^{\circ}$ and $\Delta \beta=3.6^{\circ}$ for $h k l=(100),(110),(111)$ and (311), also in the approximation $l_{\max }=34$. These pole figures were then used as 'experimental' starting data for the following calculations. They correspond exactly with the above mentioned theoretical texture, whereby no truncation error occurs and no odd part needs to be considered. The so defined 'experimental' pole figures are exactly normalized which allows an easy check for the obtained normalization factors $N_{h k l}$.

\section{Fibre Textures}

In the case of fibre textures, the integration over $\beta$, eq. 8 , is trivial. Fibre textures are thus very well suited to test the interpolation and extrapolation in $\alpha$ according to eq. 9 and eq. 12-14. 
Complete pole figures If the calculated pole figures are used in the complete range $0 \leqslant \alpha \leqslant 90^{\circ}$, the coefficients $F_{l}^{v}(h k l)$ can be calculated according to eq. 9. The normalization factors are then obtained from eq. 10. They are given in Table I.

TABLE I

Normalization factors $N_{h k l}$ obtained from complete pole figures of two (100)-fibre textures by the spline interpolation

\begin{tabular}{ccc}
\hline & \multicolumn{2}{c}{$\omega_{0}$} \\
& $7.5^{\circ}$ & $12.5^{\circ}$ \\
\hline$(100)$ & 1.002 & 1.000 \\
$(110)$ & 1.001 & 1.000 \\
$(111)$ & 1.000 & 1.000 \\
$(311)$ & 1.001 & 1.000 \\
\hline
\end{tabular}

The deviation from the ideal value 1 is less then $10^{-3}$ in the case of the smoother texture with $12.5^{\circ}$ spread and in the order $2.10^{-3}$ for the sharper texture. Table $I$ is thus a measure of the error due to the finite steps $\Delta \alpha$ using spline interpolation.

The textures themselves were then calculated by solving eq. 2 for the coefficients $C_{l}^{\mu 0}$ and using eq. 1 with $l_{\max }=34$. In the case of fibre textures the ODF is independent of the angle $\varphi_{1}$. It is equivalent to the inverse pole figure of the fibre axis which exhibits its maximum value at the orientation (100). These maximum values are very sensitive to calculation errors. Hence, they provide a good test of the accuracy of the calculation. In Table II the maximum values obtained by spline interpolation are given for the two textures.

TABLE II

\begin{tabular}{|c|c|c|}
\hline & & \\
\hline & $7.5^{\circ}$ & $12.5^{\circ}$ \\
\hline theor. & 38.8 & 14.09 \\
\hline linear & 34.1 & 13.40 \\
\hline spline & 37.9 & 14.08 \\
\hline
\end{tabular}

They are compared with theoretical values obtained directly from eq. 15 and eq. 1 (with $l=$ even and $l_{\max }=34$ ) and with the corresponding values obtained with a linear interpolation instead of the spline interpolation of eq. 9. In the case of the smoother texture with 
$\omega_{0}=12.5^{\circ}$, the deviation between the theoretical value and the one obtained by spline interpolation is less than $10^{-3}$ whereas linear interpolation introduces an error of $5 \%$. In the case of $\omega_{0}=7.5^{\circ}$, the improvement due to the spline interpolation is not so strong, but at least the error is reduced from $12 \%$ to $2 \%$.

Incomplete pole figures By omitting part of the pole figures obtained with the coefficients of eq. 15, incomplete "experimental" pole figures were obtained. The extrapolation of these pole figures into the unknown range $\alpha>\alpha_{\max }$ obtained by eqs. 12-14 is shown in Figs. 1a-d for the sharper texture with $\omega_{0}=7.5^{\circ}$. In most cases, the extrapolated function fits the experimental one reasonably well. Only in the case of the (311)-pole figure truncated at $\alpha_{\max }=60^{\circ}$, the available information is insufficient. In the case of the smoother texture $\omega_{0}=12.5^{\circ}$, the fit is of course better. The extrapolated functions are only used in order to calculate the normalization factors, hence, the obtained approximation is quite satisfactory.

The normalization factors $N_{h k l}$ as a function of the truncation angle $\alpha_{\max }$ are given in Figs. 2a-d. It is seen that the deviation of the calculated values is less than $1 \%$ up to $\alpha_{\max }=70^{\circ}$ even for the sharp texture with $\omega_{0}=7.5^{\circ}$. At $\alpha_{\max }=60^{\circ}$ the deviation increases but it still remains within about $10 \%$ even in the case of the (311)-pole figure of the sharp texture.

With these normalization factors, the ODF was then calculated according to eq. 11 and eq. 1 . The deviation of the maximum density value at (100) from the one obtained from complete pole figures is shown in Fig. 3 for the two textures. The deviation inreases to about $5 \%$ at $\alpha_{\max }=60^{\circ}$ for the sharp texture. This seems to be tolerable, since most of the experimental pole figure measurements are obtained with an experimental error in the same order of magnitude.

\section{Cube Textures}

Cube textures with $\omega_{0}=7.5^{\circ}$ and $\omega_{0}=12.5^{\circ}$ were calculated in the same way using eq. 15 . In this case also the integration over $\beta$ according to eq. 8 may introduce an error.

Complete pole figures Spline interpolation in $\alpha$ was carried out according to eq. 9. Again the normalization factor was obtained by eq. 10. It is given in Table III. 


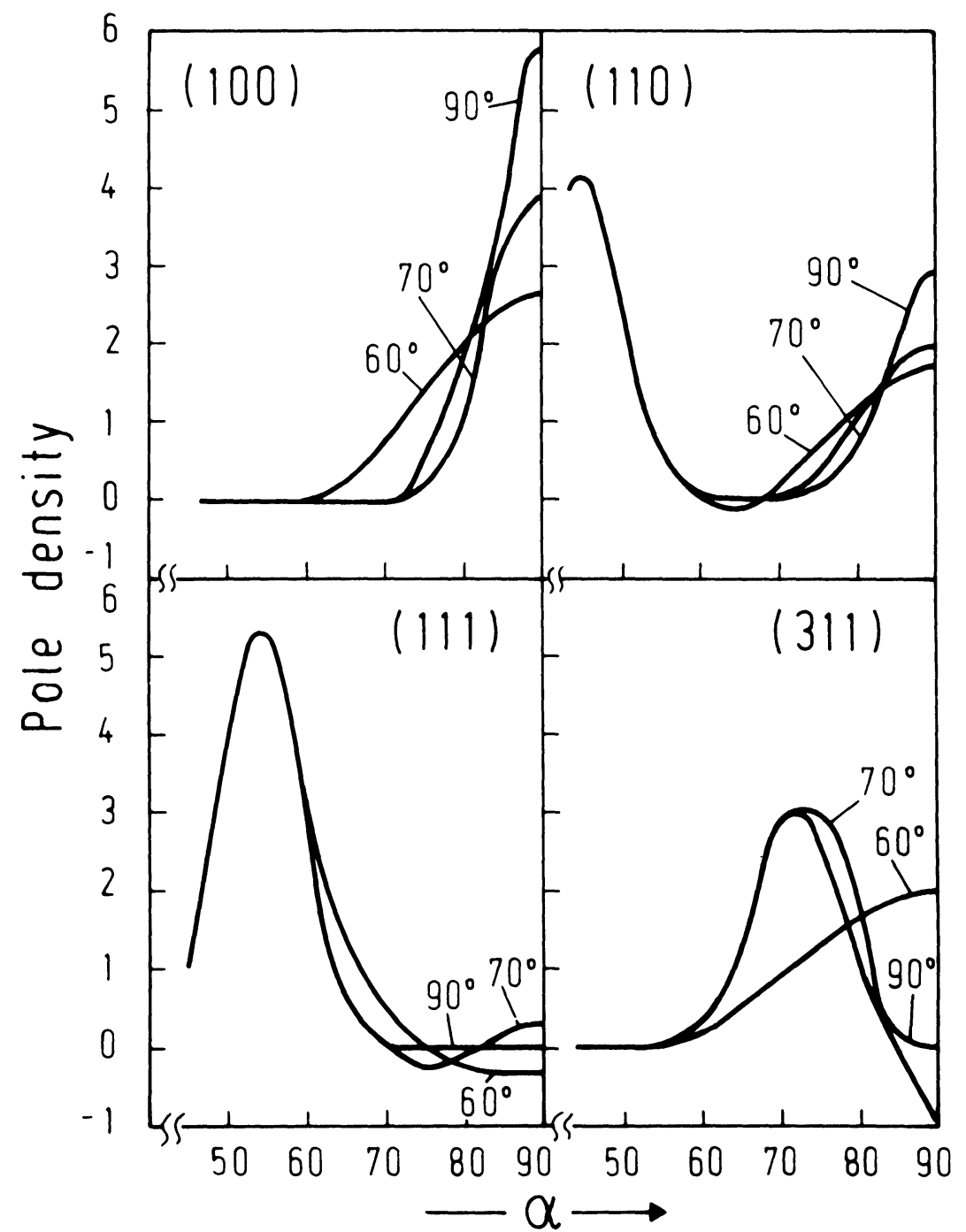

FIGURE 1 Extrapolated pole figures of a (100)-fibre texture with $\omega_{0}=7.5^{\circ}$ for truncation angles $\alpha_{\max }=60^{\circ}$ and $70^{\circ}$, compared with the original ones $\left(\alpha_{\max }=90^{\circ}\right)$. 
TABLE III

Normalization factors $N_{h k l}$ for complete pole figures of two different cube textures obtained by spline interpolation

\begin{tabular}{ccc}
\hline & & $\omega_{0}$ \\
& $7.5^{\circ}$ & $12.5^{\circ}$ \\
\hline$(100)$ & 1.002 & 0.998 \\
$(110)$ & 1.001 & 1.000 \\
$(111)$ & 1.000 & 1.000 \\
$(311)$ & 0.998 & 0.999 \\
\hline
\end{tabular}

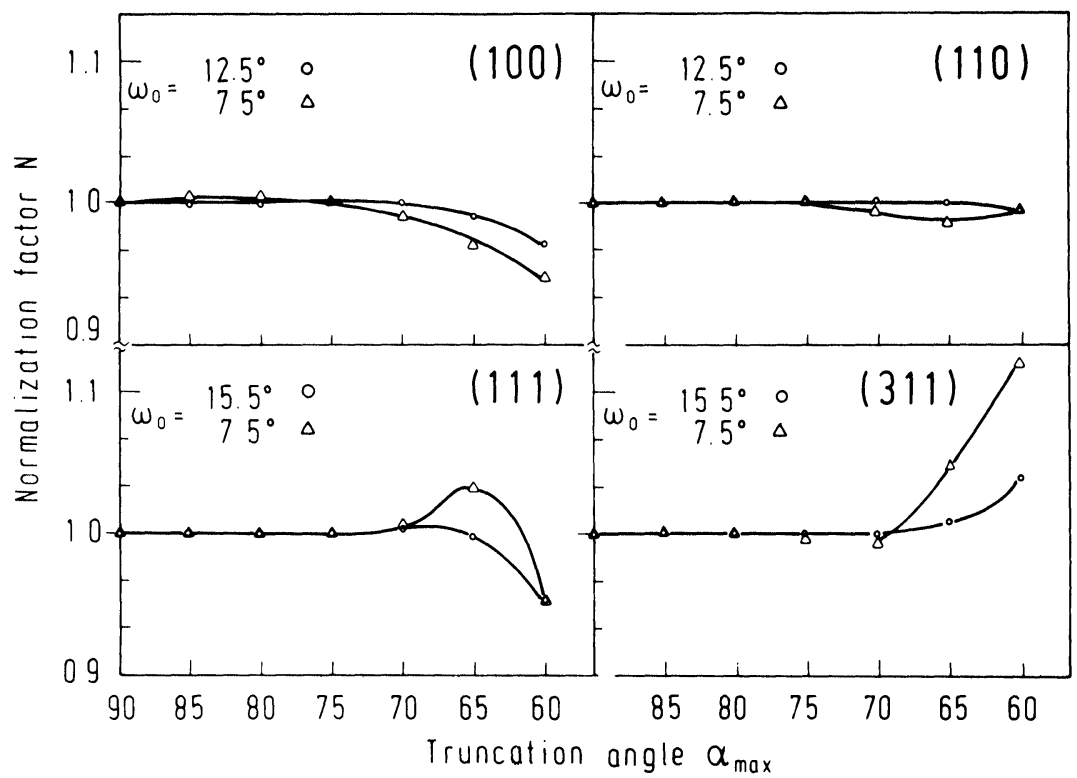

FIGURE 2 Normalization factors of two (100)-fibre textures as a function of the truncation angle $\alpha_{\max }$.

The results are similar to the ones given in Table I, the differences being due to the numerical integration in $\beta$, eq. 8 . The ODFs themselves were obtained by eq. 9, 2 and 1 . The maximum values at the ideal cube position are given in Table IV. 


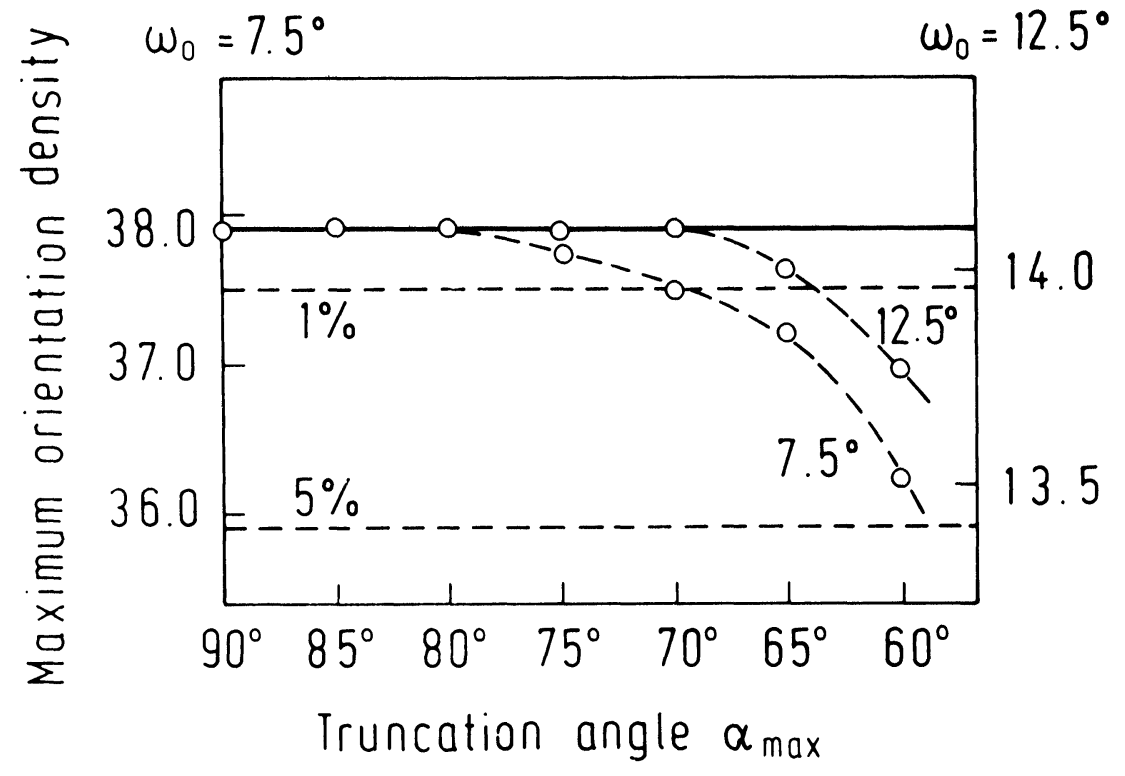

FIGURE 3 Maximum density values of the ODF (inverse pole figure of the fibre axis) of two (100)-fibre textures determined from incomplete pole figures as a function of the truncation angle $\alpha_{\max }$.

TABLE IV

Maximum values $f_{\max }$ of the ODF for two cube textures

\begin{tabular}{lccc}
\hline & $7.5^{\circ}$ & $\omega_{0}$ & \\
& & $12.5^{\circ}$ \\
\hline theor. & 173.4 & & 44.0 \\
linear & 155.7 & & 42.2 \\
spline & 164.4 & & 43.4 \\
\hline
\end{tabular}

It is seen that the deviation from the theoretical texture is about $1.4 \%$ for the smoother texture whereas linear interpolation introduces about $4 \%$ error. For the sharper texture, the error is reduced from $10 \%$ in the case of linear interpolation to $5 \%$ in the case of spline interpolation.

Incomplete pole figures As in the case of fibre textures, incomplete "experimental" pole figures were obtained by omitting part of the values of the pole figures calculated from the coefficients of eq. 15. The 
normalization factors were obtained by extrapolating $S_{h k l}^{0}(\alpha) \sin \alpha$ into the omitted range $\alpha>\alpha_{\max }$. The ODF was then obtained from eq. 11 and eq. 1 . In this case the reliability of the obtained results was checked by calculating the texture $J=\Sigma_{l \mu v}\left[C_{l}^{\mu \nu}\right]^{2}$ which is shown in Fig. 4 as a function of the truncation angle $\alpha_{\max }$.

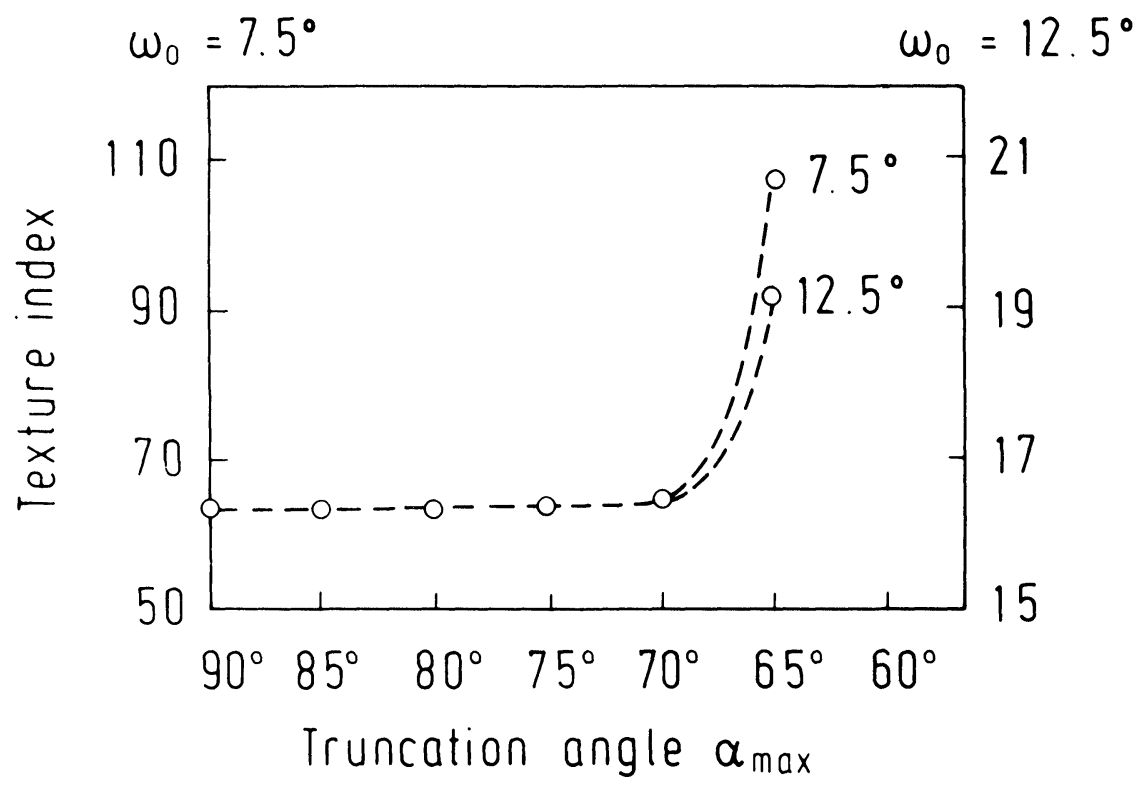

FIGURE 4 The texture index $\mathrm{J}$ for two cube textures calculated from incomplete pole figures as a function of the truncation angle $\alpha_{\max }$.

It is seen that up to $\alpha_{\max }=70^{\circ}$ there is nearly no loss of information introduced by the truncation. The increase of the texture index at lower limiting tilt angles is not only due to errors in the normalization factors but also due to errors introduced by solving eq. 11 as has been shown for instance by Pospiech and Jura (1974).

\section{CONCLUSIONS}

The error introduced by numerical integration over a pole figure which is available only at discrete points $\left(\alpha_{i} \beta_{j}\right)$ can be reduced by taking into 
account that neighbouring pole density values are not completely independent of each other since they belong to a function of "limited slope". Spline interpolation is an "economic" way to take the slope of an experimental function into account, and spline programs are available in most software systems. The obtained results show that with steps of $3.6^{\circ}$ and $5^{\circ}$ respectively, as they are usual in pole figure measurements, the integration errors can be kept considerably below $1 \%$ even with relatively sharp textures. This must be considered sufficient compared to the usual experimental error of measured pole figures.

Consideration of the slope of pole density curves also allows an improved extrapolation into the unknown range of incompletely measured pole figures. Errors of the order of $1 \%$ for a $70^{\circ}$ truncation or $5 \%$ even for a $60 \%$ truncation in the case of a relatively sharp texture are quite satisfactory compared with the experimental errors of pole figure measurement.

\section{Acknowledgement}

M. Dahms wishes to thank P. I. Welch for useful discussions in the early stages of the work.

\section{References}

Bunge, H.-J. Mathematische Methoden der Texturanalyse, Akademie Verlag Berlin (1969).

Van Houtte, P. Mat. Sci. Eng. 43, 7-11 (1980).

Van Houtte, P. Textures and Microstructures 6, 137-161 (1984).

Humbert, M. \& Bergmann, H.W. J. Appl. Cryst. 13, 500-504 (1980).

Jordan-Engeln, G. \& Reutter, F. Formelsammlung zur Numerischen Mathematik mit Standard-FORTRAN-Programmen, Bibliographisches Institut Mannheim (1981).

Kern, R. \& Bergmann, H. W. In: Textures of Materials, Eds. G. Gottstein and K. Lücke, Springer Verlag Berlin (1979).

Liang, Z., Xu, J. \& Wang, F. Proc. ICOTOM 6, 1259-1264 Tokyo (1981).

Liang, Z., Xu, J. \& Wang, F. Mat. Sci. Eng. 60, 59-63 (1983).

Morris, P. R. Adv. X-ray Anal. 18, 514-534 (1975).

Pospiech, J. \& Jura, J. Z. Metallkunde 65, 324-330 (1974). 